\title{
A single-camera, 3D scanning velocimetry system for quantifying active particle aggregations
}

\author{
Matt K. Fu • Isabel A. Houghton • John O. Dabiri
}

Received: date / Accepted: date

\begin{abstract}
A three-dimensional (3D) scanning velocimetry system is developed to quantify the 3D configurations of particles and their surrounding volumetric, three-component velocity fields. The approach uses a translating laser sheet to rapidly scan through a volume of interest and sequentially illuminate slices of the flow containing both tracers seeded in the fluid and particles comprising the aggregation of interest. These image slices are captured by a single high-speed camera, encoding information about the third spatial dimension within the image time-series. Where previous implementations of scanning systems have been developed for either volumetric flow quantification or 3D object reconstruction, we evaluate the feasibility of accomplishing these tasks concurrently with a single-camera, which can streamline data collection and analysis. The capability of the system was characterized using a study of induced vertical migrations of millimeter-scale brine shrimp (Artemia salina). Identification and reconstruction of individual swimmer bodies and 3D trajectories within the migrating aggregation were achieved up to the maximum number density studied presently, $8 \times 10^{5}$ animals per $\mathrm{m}^{3}$. This number density is comparable to the densities of previous depth-averaged 2D measurements of similar migrations. Corresponding velocity measurements of the flow indicate that the technique is capable of resolving the 3D velocity field in and around the swimming aggregation. At these animal number densities, instances of coherent flow induced by the migrations were observed. The accuracy of these flow measurements was confirmed in separate studies of a free jet at $R e_{D}=50$.
\end{abstract}

Keywords First keyword · Second keyword · More

\section{Introduction}

Turbulent flows containing dispersed particles are a common feature in many environmental and industrial processes. The particles within these flows include both passive phases such as solid particles (Balachandar and Eaton, 2010), bubbles (Rensen et al., 2005, Risso, 2018), and droplets (Aliseda and Heindel, 2021), as well as 'active' phases such as swimming zooplankton (Jumars et al., 2009). At sufficiently large particle volume fractions $\left(10^{-6} \leq \Phi_{v} \leq 10^{-3}\right)$, the presence of the particles creates unique flow dynamics associated with the

Funding for this project was generously provided by the Gordon and Betty Moore Foundation

M. K. Fu and J. O. Dabiri

California Institute of Technology

GALCIT

1200 E California Blvd.

MC 105-50

Pasadena, CA 91125

E-mail: mkfu@alumni.princeton.edu

I. A. Houghton

Sofar Ocean

Pier 50, Shed B, Bulkhead Office

San Francisco, CA 94158 
two-way fluid-particle coupling that are distinct from single-phase turbulence (Elghobashi, 1994). Characterizing this two-way coupling requires accurately reconstructing the three-dimensional (3D) aggregations of particles and the turbulent flow field in which they are dispersed. Because the fluid-particle interactions are 3D and occur over a wide range of spatiotemporal scales, there are many challenges to measuring them experimentally. These challenges are exacerbated in denser aggregations where there are larger numbers of particles and interactions that need to be tracked and quantified (Bourgoin and $\mathrm{Xu}, 2014$ ).

Biologically generated turbulence is an emerging topic whose study is currently limited by an inability to quantify the flow within aggregations of swimming plankton. The turbulence created by these aggregations remains a poorly understood, and potentially underrepresented, source of scalar transport and ocean mixing (Kunze, 2019). Though the eddies created by an isolated swimmer are comparable to that of the individual organism, the larger length scales associated with the aggregations of swimmers have the potential to introduce mixing scales relevant to the surrounding water column. Recent laboratory studies of millimeter-scale brine shrimp (Artemia salina) aggregations using two-dimensional (2D) flow measurement techniques have shown that induced migrations could generate aggregation-scale mixing eddies through a Kelvin-Helmholtz instability (Wilhelmus and Dabiri, 2014) with effective turbulent diffusivities several orders of magnitude larger than molecular diffusion alone (Houghton et al., 2018: Houghton and Dabiri, 2019). Though the potential for enhanced mixing is substantial, direct measurements of enhanced turbulent dissipation and mixing in lakes and the ocean due to vertical migrations have been less conclusive (Noss and Lorke, 2014, Simoncelli et al., 2018, Kunze, 2019). Parameterizing the precise conditions and mechanisms that lead to enhanced mixing remains an active area of research (Wang and Ardekani, 2012, 2015; Ouillon et al., 2020; More and Ardekani, 2021).

There are numerous efforts to develop volumetric velocimetry techniques capable of resolving the unsteady flow field in addition to the morphology and kinematics of a single swimming organism. A common technique for volumetric, three-component (3D-3C) velocity measurements is tomographic particle image velocimetry (Tomo-PIV), which has been used extensively for investigations of aquatic locomotion, including the propulsive mechanisms of fish (Gemmell et al. 2019) and pteropods (Adhikari et al., 2016). A key requirement for TomoPIV is employing four or more cameras to provide sufficient viewing angles for the tomographic reconstruction of both tracer particles used for flow quantification and swimmer bodies. Though there have been significant advancements in the resolution of Tomo-PIV for velocity quantification, most notably, through the 'Shakethe-Box algorithm' of Schanz et al. (2016), accurately reconstructing active or passive particles with complex, three-dimensional shapes remains challenging. One common approach to body reconstruction is to compute a visual hull based on the projection of an object onto multiple camera viewpoints (Adhikari and Longmire, 2012). This method can overestimate the body size and obscure complex or rounded body geometries. While these shortcomings can be moderated by prescribing additional constraints to the body morphology or kinematics, such an approach typically requires a priori knowledge of the behavior of the dispersed phase (Ullah et al., 2019). Despite these advancements, accurately reconstructing dense aggregations of particles, especially those with complex morphology, remains elusive.

Beyond Tomo-PIV, several alternative 3D-3C techniques have been proposed for marine swimming quantification, including plenoptic imaging (Tan et al., 2020), synthetic aperture particle image velocimetry (Mendelson and Techet, 2015, 2018), defocusing digital particle image velocimetry (DDPIV) (Pereira and Gharib, 2002; Troutman and Dabiri, 2018), and 3D digital holography (Gemmell et al., 2013). Though all of these techniques have been demonstrated on individual swimmers, few are suitable for object reconstruction, and none have been successfully deployed to reconstruct dense configurations of swimmers and tracer particles in 3D.

Here, we present a 3D scanning system to reconstruct configurations of vertically migrating swimmers and quantify their surrounding 3D-3C velocity field. Several scanning systems have been developed in recent years for a variety of applications, including 3D-3C velocity measurements (Hoyer et al., 2005, Brücker et al., 2013, Lawson and Dawson, 2014; Ni et al., 2015, Kozul et al., 2019) and 3D object reconstruction of translucent organisms (Katija et al. , 2017, 2020) and structures (Su et al., 2018). The 3D scanning system in the present study is conceptually similar to those existing systems but is used to simultaneously quantify the locations and organizations of the swimmers and their surrounding flow field. The approach relies on a laser sheet that rapidly and repeatedly scans through a volume of interest, sequentially illuminating image slices of flow tracer particles and organism cross-sections. The images are captured by a single high-speed camera, encoding detailed information about the third spatial dimension within the image time-series. Repeated scanning creates a series of image volumes consisting of swimmer bodies and tracer particles. Due to their large size relative to the tracer particles, the swimmer bodies can be identified and tracked over time. Similarly, the velocity field in the vicinity of the swimmers is determined via localized $3 \mathrm{D}$ cross-correlations of consecutive tracer particle images. 
The capabilities of the technique are demonstrated by scanning induced vertical migrations of brine shrimp (Artemia salina). We demonstrate that the 3D position, orientation, and morphology of individual A. salina can be faithfully reconstructed, even at large animal number densities up to $8 \times 10^{5}$ animals per $\mathrm{m}^{3}$, the high end of previously reported brine shrimp number densities in the literature (Houghton and Dabiri, 2019). We then show selected examples in which a coherent, large-scale induced flow is resolved by the measurement technique. While the appearance of large-scale induced flow was not observed during each migration, the present results demonstrate the ability of the measurement technique to capture those dynamics when they do occur. Lastly, the outlook for the technique is discussed with suggested technical improvements to the system design.

\title{
2 Scanning 3D Image Reconstruction System
}

\author{
2.1 Imaging Hardware and Procedure
}

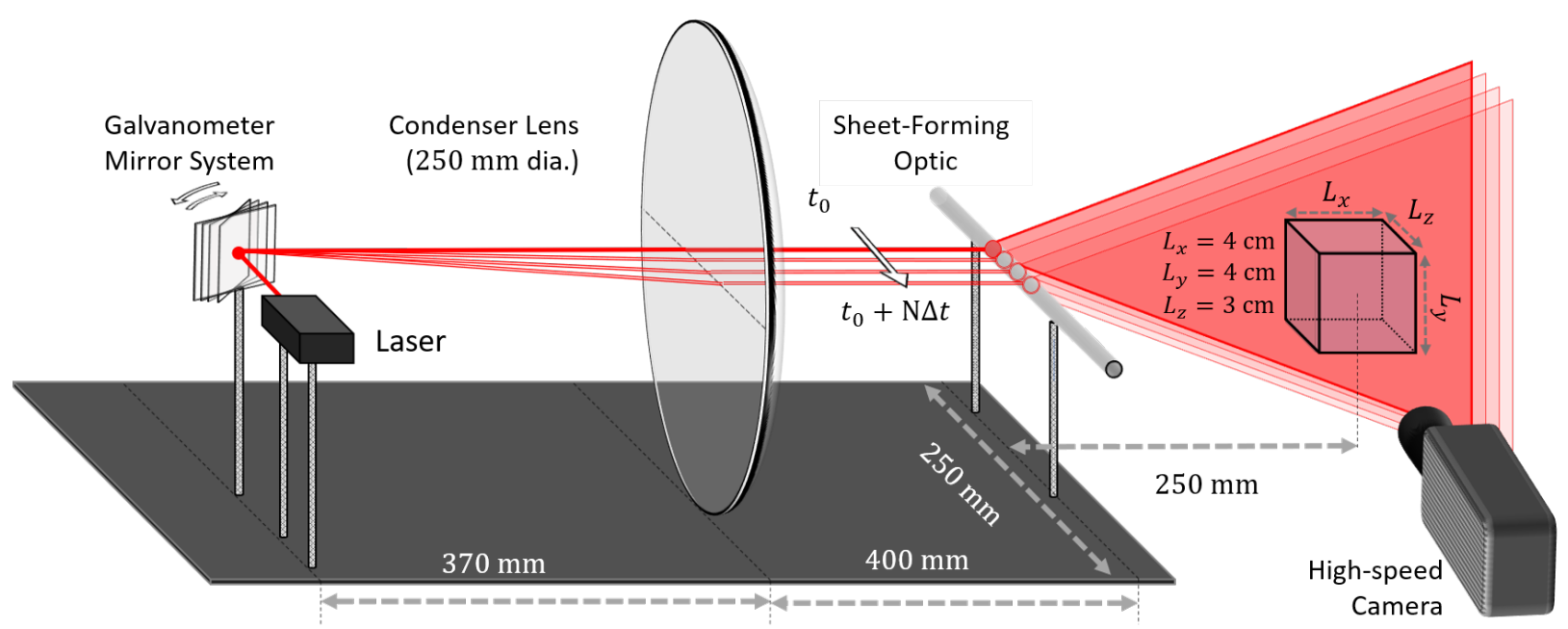

Fig. 1: Diagram of the scanning system. A mirror-mounted galvanometer (left) deflected the imaging laser along the scanning direction. A condenser lens (center-left) collected the angled beams to create displaced but parallel beams. The beams were then formed into scanning sheets by a long sheet forming optic (center-right). The imaging volume (right) was repeatedly scanned to sequentially illuminate slices of tracers and particles. The image slices were captured by a high-speed camera (lower-right) and then stacked to form 3D image volumes.

The design of the scanning system, shown in Figure 1 was similar to the system of Lawson and Dawson (2014). Illumination for the scanning was provided by a $671 \mathrm{~nm}$ continuous wave laser (5-Watt Laserglow LRS0671 DPSS Laser System). This wavelength of light ensured that the brine shrimp exhibited no phototactic response to the imaging light. Additionally, the laser beam had only a single Transverse Electric Mode (i.e., near $\mathrm{TEM}_{00}$ or quasi-Gaussian beam) to minimize imaging artifacts along the scanning dimension due to the beam shape.

The laser beam was angled along the scanning dimension of the imaging volume by a mirror with a broadband dielectric coating (-E02) mounted on a single-axis galvanometer (Thorlabs GVS211/M). The angular range $\left(\max \pm 20^{\circ}\right)$ and bandwidth $(65 \mathrm{~Hz}$ square wave at $50 \%$ full travel) of the galvanometer were comparable to other scanning systems in the literature that rely on scanning optics such as rotating polygonal mirrors (Hoyer et al. 2005, Brücker et al., 2013) or piezo-electric mirrors (Ni et al., 2015). An analog voltage signal from an arbitrary function generator (Tektronix AFG3011C) controlled the tilt of the mirror, which determined the position and scanning rate of the laser. The angled beams were collected by a $250 \mathrm{~mm}$ dia. condenser lens (370 mm back focal length), realigning them into parallel trajectories displaced along the scanning direction. These scanning beams were then converted into scanning sheets by a sheet forming optic that spans the depth of the imaging volume, such as a glass cylinder. The size of the condenser lens and the length of the sheet 
forming optic determined the maximum distance over which the beams could be collected and aligned. By employing a condenser lens with a relatively large focal length, the amount of mirror rotation necessary to deflect the beams over the entire depth of field was contained to just a few degrees $\left( \pm 1.2^{\circ}\right.$ in the present study). Here, the galvanometer was driven with a sawtooth wave to repeatedly scan the imaging volume with a constant forward scanning speed that filled approximately $94 \%$ of the scanning period. The remaining $6 \%$ of the scanning period was spent on the backward scan to reset the mirror position for the next imaging period. The accuracy of the scanning rate was limited by the repeatability of the galvanometer $(0.07 \%$ for $30 \mu \mathrm{rad}$ beam angle repeatability).

By rapidly scanning a laser sheet along the sheet-normal axis, 1 millimeter-thick image slices throughout the depth of the interrogation volume were sequentially illuminated and captured by a high-speed camera. By ensuring that the scanning period was considerably faster than the flow time scales (e.g. laser translation speed 30 times faster than the animal swimming speed in the present experiments), the recorded images could encode spatial information about the scanning dimension within the image time-series. The image sequences were stacked to construct volumetric (3D) images of the quasi-static tracers and larger active or passive particles, such as the swimmers of present interest. Periodic scanning of the interrogation volume facilitated tracking of the particles and tracers over time.

\subsection{Imaging Acquisition \& Calibration}

The scanned images were captured with a high-speed camera (Photron FASTCAM SA-Z) equipped with a fixed focal length macro lens (Micro-NIKKOR $105 \mathrm{~mm}$ with a $36 \mathrm{~mm}$ extension tube) at $1024 \times 1024 \mathrm{px}^{2}$ resolution. The image acquisition rate was matched to the scanning speed such that the displacement of the laser sheet between each frame was approximately the same size as the mean pixel resolution (i.e., $40 \mu \mathrm{m}$ ). This fine depth-wise sampling allowed the raw image volume to have a nearly isotropic voxel size. Both the f-number $(f / 22)$ and working distance (approximately $0.4-0.5 \mathrm{~m})$ were iteratively tuned to ensure that the entirety of the imaging volume was within the depth of field $(3 \mathrm{~cm})$ and each scanned image was in sharp focus.

A custom 3D calibration target (UCrystal) was fabricated to calibrate the imaging volume and account for the 7\% change in magnification along the scanning depth. The target, shown in Figure 2a, comprised an $8 \mathrm{~cm} \times 8 \mathrm{~cm} \times 8 \mathrm{~cm}$ crystal cube internally laser engraved with a 3-dimensional grid of $1.6 \mathrm{~mm}$ diameter spherical shells. The shells were evenly spaced $1 \mathrm{~cm}$ apart in each direction to form a $6 \times 6 \times 6$ cubic array $(5 \mathrm{~cm} \times 5 \mathrm{~cm} \times 5 \mathrm{~cm})$, which was centered within the crystal. The spot size of the laser engraver used to raster the spherical shells was approximately $100 \mu \mathrm{m}$. The cube was suspended at the center of the imaging volume and aligned with the imaging coordinate system to ensure that the laser sheet was not deflected by refraction inside the cube.

Calibrating the imaging system involved scanning the calibration cube with the laser sheet and capturing the 2-D image slices with the high-speed camera. The images collected over each period were stacked to form a single 3-D image volume. Because the scanning was designed to create nearly isotropic volumes, minimal processing of out-of-plane dimension was necessary to render scanned objects.

The raw image volumes were processed and analyzed using MATLAB's Image Processing Toolbox to reconstruct and locate the spherical targets. The image volume was median filtered ( $7^{3}$ vx. stencil) and binarized with a global threshold based on the image histogram. Morphological area opening was then used to remove objects other than the calibration spheres, e.g., tracer particles and camera noise, from the binary image, leaving just the calibration spheres. Any holes within the binary images of the spheres were then filled. The centroids of the remaining spheres (shown in Figure 2b) were then used to calibrate the image volume. While all of the target spheres were scanned, not all of them were successfully reconstructed. This failure was most common in target spheres further from the camera as their scattered light could be obstructed by spheres in the foreground.

By relating the centroids of the rendered spheres to the known dimensions of the calibration target, the voxels within the image volume could be mapped to 3D coordinates in physical space. The mapping between the two coordinate systems was calculated using the MATLAB estimateCameraParameters function.

\subsection{Particle Segmentation}

Just as the spheres were extracted from the calibration target, we do the same for the active/passive particles in an aggregation. Because the particles in this study, i.e., the swimmers, were significantly larger than the tracers, they could be identified and segmented within the image volume by size. This segmentation process was 


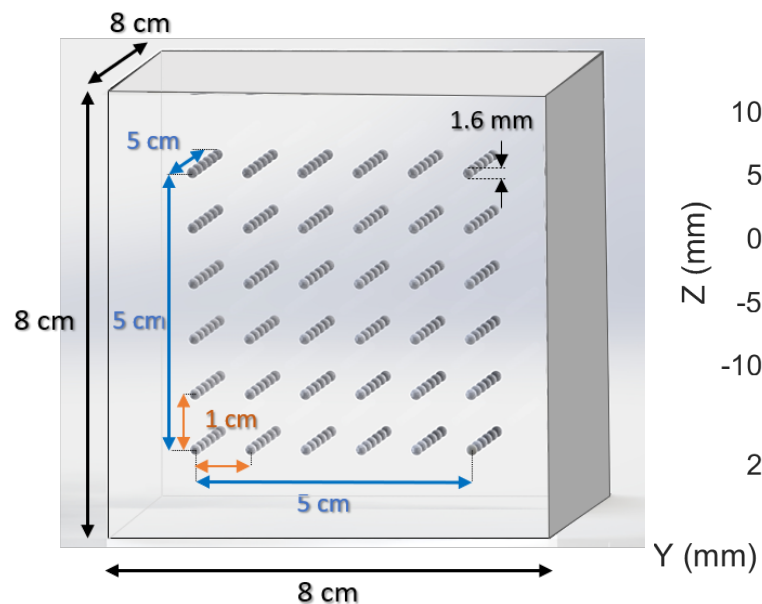

(a)

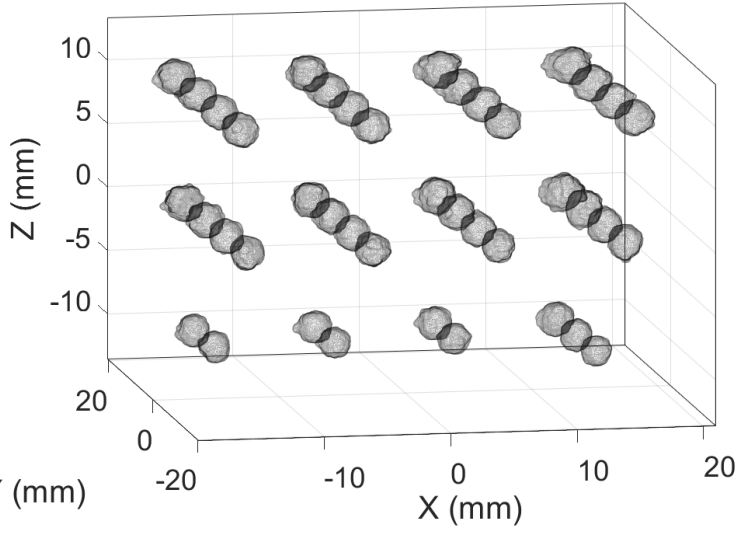

(b)

Fig. 2: Images of the calibration cube target. (a) CAD Rendering of the $3 \mathrm{D}$ calibration cube. The $6 \times 6 \times 6$ array of spheres can be seen in the center of the $8 \times 8 \times 8 \mathrm{~cm}^{3}$ cube. (b) Rendered scan of the calibration cube from the $3 \mathrm{D}$ scanning system. Some spheres are not reconstructed in the image due to low scattering intensity.

accomplished by filtering the raw images with a cubic Gaussian kernel (3 vx. stencil). The filtered images were then binarized with the method of Otsu (Otsu, 1979), which computes a global threshold based on the image histogram. Tracers were removed from the binary image by filtering out objects smaller than 8000 connected voxels through morphological area opening. This 8000-voxel threshold was found to work satisfactorily for the specific imaging parameters in this study. Depending on the application and object size distribution, alternative segmentation techniques, such as the 3D analogs of those reviewed by Khalitov and Longmire $(2002)$, may prove more robust. Connected components within the binary image were labeled as individual swimmer bodies. The centroids of each of the swimmer bodies were tracked over time to determine the swimmer trajectories. A mask for the tracer field was computed by morphologically dilating the binary image of the particles with a spherical structuring element ( 4 vx. radius).

\subsection{Velocity Field Registration}

With the particles comprising the aggregation segmented, the remainder of the image corresponding to the tracer field was then used to compute the volumetric, three-component velocity field by registering the local displacements of tracer particles between successive images. Each pair of image volumes was first masked using the binary images of swimmer bodies from the previous segmentation step. Each mask was applied to both images in the pair to ensure each frame had an identical mask and avoid correlations due to mask shifting.

To resolve the local tracer displacement between consecutive images, we employed a modified version of the Fast Iterative Digital Volume Correlation (FIDVC) Algorithm of Bar-Kochba et al. (2015). This method could resolve large volumetric deformations between two images by conducting 3D cross - correlations on progressively refined interrogation windows to compute the local image displacement. First, the original images were divided into $64 \times 64 \times 64 \mathrm{vx}^{3}$ windows with $50 \%$ overlap. Each windowed image was weighted with the modular transfer function of Nogueira et al. (2005) to stabilize the spatial frequency content. The 3D, voxel displacement between the two images was determined to the nearest integer voxel by finding the local maximum of the cross-correlation function between the two windows. Sub-voxel resolution for the displacement was then achieved by first conducting a least-squares fit with a 3D Gaussian function to the $5^{3}$ voxel neighborhood around the peak value in the cross-correlation function. The sub-voxel displacement was then determined by solving for the local maximum of the resulting fit.

Displacement vectors with correlation coefficients below a certain threshold $(\leq 0.01 \%$ of the maximum correlation) or within the image mask were rejected and replaced with interpolated values. The displacement field was then filtered with the tunable low pass convolution filter of Schrijer and Scarano (2008) to improve the 
iterative image deformation, and all nonphysical outliers were removed via a universal median test (Westerweel and Scarano, 2005). Both image volumes were then symmetrically deformed by a tri-cubic interpolation scheme using the MATLAB griddedInterpolant function. The root mean square (RMS) deviations between the two images before and after deformation were computed and their ratio was used as a convergence metric. When the RMS deviation ratio was reduced to less than 0.1, the window size was refined for the next iteration. The iterative deformation process was repeated until the minimum window size $\left(32 \times 32 \times 32 \mathrm{vx}^{3}\right.$ with $75 \%$ overlap) was reached and the final RMS ratio was less than 0.2 . These convergence criteria were found to provide an acceptable balance between accuracy and computation times for the images analyzed in this study and typically required 7 iterations to achieve convergence. All Fast Fourier Transforms (FFTs) and sub-voxel estimation operations were executed with the MATLAB Parallel Computing Toolbox on two NVIDIA Quadro RTX5000 GPUs with double precision. This GPU variant was benchmarked against the original FIDVC code (Bar-Kochba et al. 2015) with agreement found in all cases up to single precision.

\section{Induced Vertical Migrations of Artermia Salina}

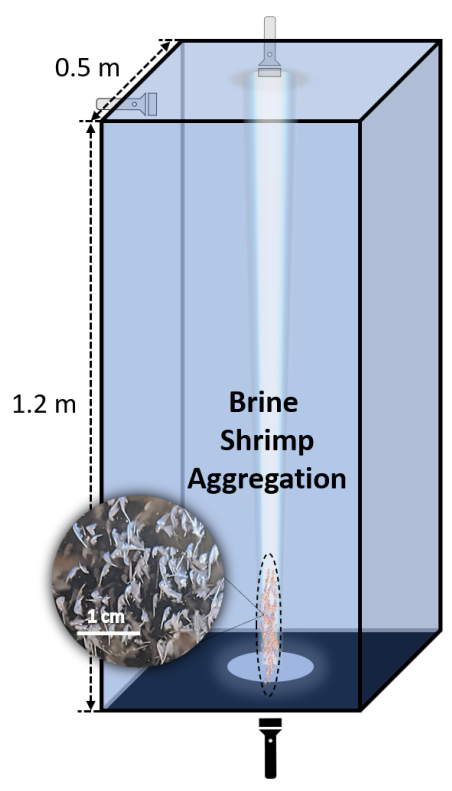

(a)

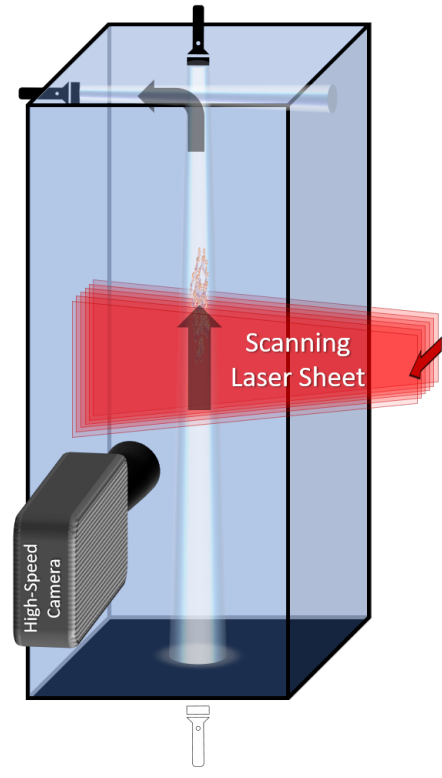

(b)

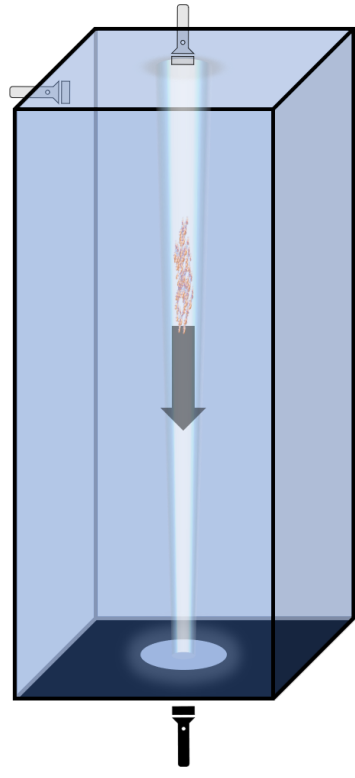

(c)

Fig. 3: Illustration of the Artemia salina vertical migration and image acquisition. (a) The brine shrimp were collected at the bottom of the $1.2 \mathrm{~m}$ tall tank with an upward projecting LED light stimulus (PeakPlus LFX1000, 600 lumens). After the Artemia salina reached the bottom, the tank was allowed to settle for at least 20 min. to ensure the fluid was quiescent. (b) An upward migration was induced with a similar downward projecting light stimulus at the top of the tank. An additional light was used to steer the brine shrimp horizontally along the free surface to the side of the tank, reducing the number of swimmers that would accumulate and obstruct the downward projecting light. The imaging volume was positioned along the central axis of the tank approximately $40 \mathrm{~cm}$ below the tank free surface. (c) A downward migration was induced with the first upward-facing light to return the shrimp to the bottom of the tank.

To test the capability of the technique in capturing aggregation kinematics and associated fluid mechanics, we evaluated vertical migrations of brine shrimp (Artemia salina) within a laboratory tank following the methodology of Houghton et al. (2018) and Houghton and Dabiri (2019) and imaged the resulting flow. This application was selected due to the challenge that the animal number density presented to existing techniques. Additionally, the slow evolution of the migration was compatible with the achievable scanning rate of the current system $(\mathcal{O}(1)$ sec). By leveraging the positive phototaxis of A. salina towards sources of blue and green 
wavelengths of light, coordinated swimming of a brine shrimp aggregation could be directed up and down the height of a 1.2-meter tall vertical tank (see Figure 3). A collection of approximately 40,000 $\pm 5,000$ animals (Northeast Brine Shrimp) was introduced to the tank for testing, corresponding to a tank-averaged abundance of 130,000 $\pm 16,000$ animals per $\mathrm{m}^{3}$. The brine shrimp had a typical body length of $5 \mathrm{~mm}$ and a nominal swimming speed of $5 \mathrm{~mm} / \mathrm{s}$. The tank was seeded with $13 \mu \mathrm{m}$ CONDUCT-O-FIL silver coated glass spheres (Potters Industries, Inc.) to facilitate imaging of the flow field.

Before the migration, the animals were collected at the bottom of the tank using an upward facing light stimulus (PeakPlus LFX1000, 600 lumens) introduced through the transparent floor of the water tank. After the animals reach the bottom of the tank, the water was allowed to equilibrate for at least 20 minutes to ensure the fluid was quiescent. Due to the slight negative buoyancy of $A$. salina, the animals were minimally active at the bottom of the tank. To trigger the upward migration, the light stimulus at the bottom of the tank was deactivated, and corresponding light stimuli at the top of the tank were activated. The first of these lights (PeakPlus LFX1000, 600 lumens) was directed down along the tank's central axis in a $5 \pm 2 \mathrm{~cm}$ diameter column and served as the primary stimulus to draw the animals up towards the free surface. A second horizontal light (PeakPlus LFX1000, 600 lumens), located just below the free-surface, steered the animals along the free surface and away from the primary stimulus to prevent them from accumulating and obstructing the migration. The duration of the vertical migration, typically 5-6 minutes, extended from the triggering of the lights until the accumulated $A$. salina began to obstruct the primary stimulus.

The 3D scanning system imaged the swimmer aggregation and tracers within in a $41 \times 41 \times 30 \mathrm{~mm}^{3}$ volume approximately $40 \mathrm{~cm}$ below the free surface. Throughout the vertical migration, scanning sequences were triggered at approximately 1-minute intervals to record a sequence of approximately 22,000 images, corresponding to a minimum of 26 image volumes over a 5 second period. The duration of the scanning sequence was limited by the size of the camera internal buffer (32 GB), and the 1-minute interval between scanning events was dictated by the time necessary to fully transfer the images to an external hard drive. Following the migration, the animals were returned to the bottom of the tank using the light stimulus under the transparent floor of the water tank. The complete imaging volume specifications and scanning parameters can be found in Table 1 .

\begin{tabular}{|c|c|c|c|}
\hline & Parameter & Symbol & Value \\
\hline 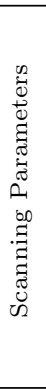 & $\begin{array}{r}\text { Field of View } \\
\text { Depth of Field } \\
\text { Approx. Voxel Size } \\
\text { Image Acq. Rate } \\
\text { Scanning Freq. } \\
\text { Scanning Speed } \\
\text { Sheet thickness } \\
\text { Sheet separation } \\
\text { Est. Seeding Density } \\
\text { No. Vols. per scan } \\
\text { Sheet step size }\end{array}$ & $\begin{array}{c}L_{x} \times L_{y} \\
L_{z} \\
f_{c} \\
f_{s} \\
u_{s} \\
h \\
\Delta z \\
N_{V} \\
\overline{\Delta z} \\
\end{array}$ & $\begin{array}{c}4 \times 41 \mathrm{~mm}^{2} \\
30 \mathrm{~mm} \\
40 \times 40 \times 40 \mu \mathrm{m}^{3} \\
4,000 \mathrm{fps} \\
5 \mathrm{~Hz} \\
15 \mathrm{~cm} \cdot \mathrm{s}^{-1} \\
1 \mathrm{~mm} \\
40 \mu \mathrm{m} \\
1.7 \times 10^{-4} \mathrm{ppv} \\
26 \\
40 \mu \mathrm{m}\end{array}$ \\
\hline 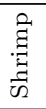 & $\begin{array}{r}\text { Body Length } \\
\text { Rel. Swimming Speed } \\
\text { Tank-Avg. No. Density }\end{array}$ & $\begin{array}{c}\ell_{c} \\
U_{s w i m} \\
n\end{array}$ & $\begin{array}{c}5-10 \mathrm{~mm} \\
3 \pm 1 \mathrm{~mm} / \mathrm{s} \\
1.3 \pm 0.16 \times 10^{5} \mathrm{~m}^{-3}\end{array}$ \\
\hline & $\begin{array}{r}\text { No. laser sheets } \\
\text { Non-dim Scan Speed } \\
\text { Non-dim Sheet Width }\end{array}$ & $\begin{array}{c}N \\
u_{s} / U_{s w i m} \\
h / \Delta z\end{array}$ & $\begin{array}{c}750 \\
30 \\
20\end{array}$ \\
\hline
\end{tabular}

Table 1: Imaging parameters for brine shrimp migrations

\section{Results}

\subsection{Body Reconstruction and Tracking}

Following the procedure outlined in section 2.3 , individual shrimp bodies were segmented in the image volume to directly assess their number, location, and orientation. A representative portion of a raw image volume is shown in Figure 4 from the camera viewpoint. Due to the translucent nature of the shrimp bodies, light was 


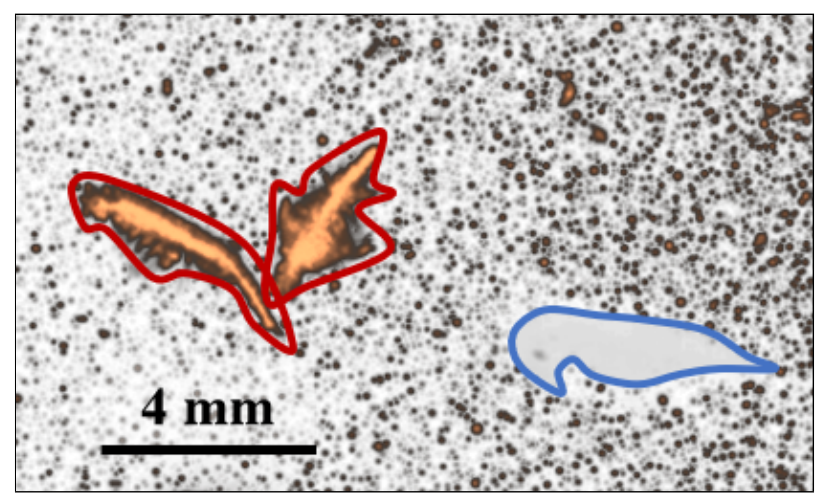

Fig. 4: Typical subsection of raw image volume of vertical migration from the camera perspective. Image intensity is inverted and colored for clarity. The shadowing effect from a foreground shrimp is outlined in blue. Reconstructed shrimp are shown in copper and outlined in red. Images of tracer particles can be seen as the dots interspersed throughout the image.

readily scattered off the organisms, allowing them to be identified as large coherent objects within the $3 \mathrm{D}$ image amongst a field of smaller tracer particles. An example of two imaged shrimp are visible in the left side of Figure 4 with a copper coloring and outlined in red for clarity. While most of the details of the shrimp morphology are evident in the image, fine features such as the shrimp legs and tail are attenuated and blurred. Due to the nature of single-camera imaging, details of the shrimp bodies and particles can be obscured or altogether blocked by objects in the foreground. An example of this shadowing effect is shown outlined in blue on the right side of Figure 4. Both the lack of visible particles and resemblance of the shadowed area to a shrimp silhouette indicated the presence of a shrimp located between the imaging volume and the camera.

The ability of the technique to reconstruct configurations of brine shrimp during a vertical migration is illustrated in Figure 5, which shows a scanned reconstruction of the animals within the full imaging volume. The approximately 40 shrimp bodies are reproduced from a scan conducted approximately four minutes into the migration and represent one of the densest collections of animals imaged during the measurement campaign. Figure 5 a shows all of the reconstructed shrimp visualized within the imaging volume. The shrimp coloring indicates their depth-wise location with positive values corresponding to locations closer to the camera. These segmented images have been corrected to account for the camera perspective and deblurred along the scanning dimension to compensate for the finite sheet thickness using Richardson-Lucy deconvolution (Biggs and Andrews, 1997). Despite the deblurring process, some elongation of the bodies in the scanning dimension was still evident. Figures $5 \mathrm{~b}-5 \mathrm{~d}$ show renderings of one animal in the migration from different viewing angles. This elongation from the scanning was most apparent in the animals tails, which appeared thicker along the scanning dimension than in the imaging plane. In the future, this effect could be mitigated through further narrowing of the laser sheet with additional optical components. Similarly, these figures also illustrate the effect of the camera perspective on the reconstruction quality. Body morphology within the line-of-sight of the camera (seen from Figure 5e) was reconstructed with higher fidelity than those obscured by the shrimp body. These differences are apparent in Figures 5 5 and $5 \mathrm{~d}$ where details such as the organisms legs were reconstructed on the right side of the organism (large values of $\mathrm{z}$ ) but were absent from the left side of the organism (smaller values of $\mathrm{z}$ ).

Even with these limitations, the reconstructed swimmers were able to capture the 3D locations, body morphology, and orientations of the scanned organisms. Though alternative single-camera techniques, such as DDPIV (Troutman and Dabiri, 2018), can similarly track particle locations in 3D, extracting a comparable level of body-specific information is neither straightforward for isolated swimmers nor possible at the high number densities present in these aggregations. Furthermore, the average animal number density measured in this scan, $8 \times 10^{5}$ animals per $\mathrm{m}^{3}$, was at the upper bound of animal number density estimates conducted in previous laboratory experiments (Houghton et al., 2018, Houghton and Dabiri, 2019). Where previous studies had to infer the animal number density during migration from depth-averaged 2D measurements, the current system was capable of measuring this quantity directly.

With the individual organisms identified, we tabulated the number of shrimp in each frame to observe the spatial and temporal evolution of the animal number density. A plot of the average number density in the imaging volume throughout four different migrations is shown in Figure 6. Reconstructions from Figure 5 correspond to the fourth minute of the first migration. Unlike previous experiments, specifically Houghton and 


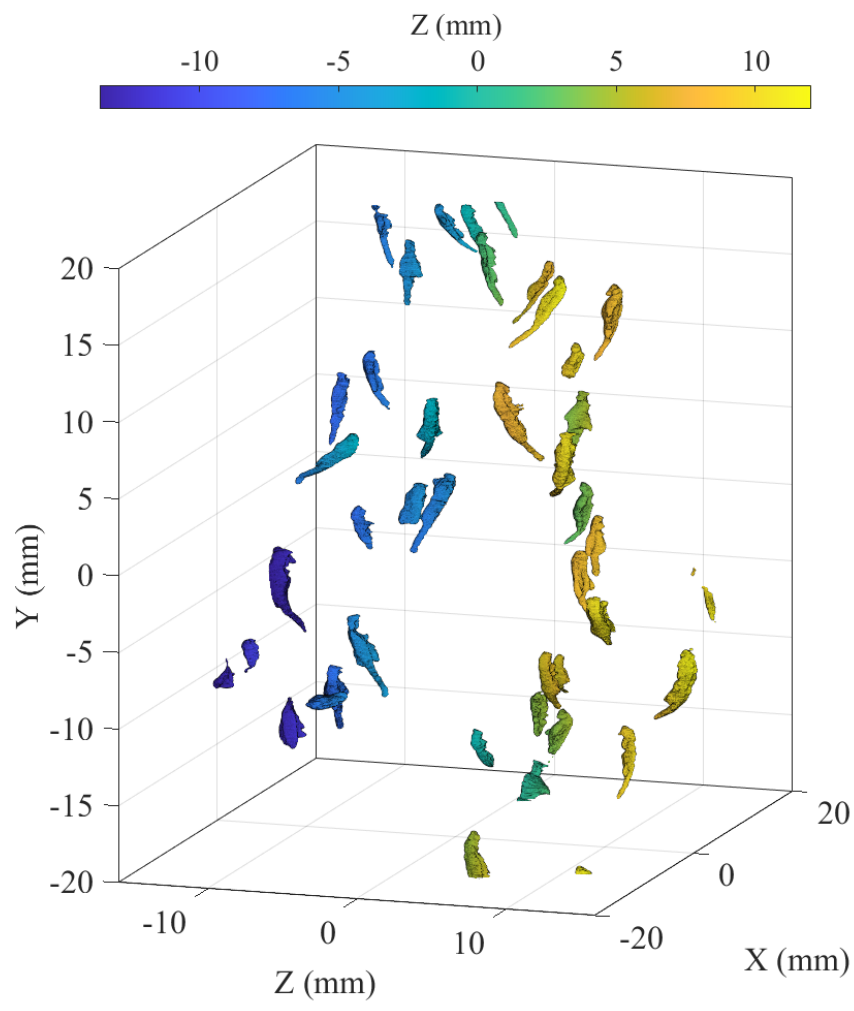

(a)
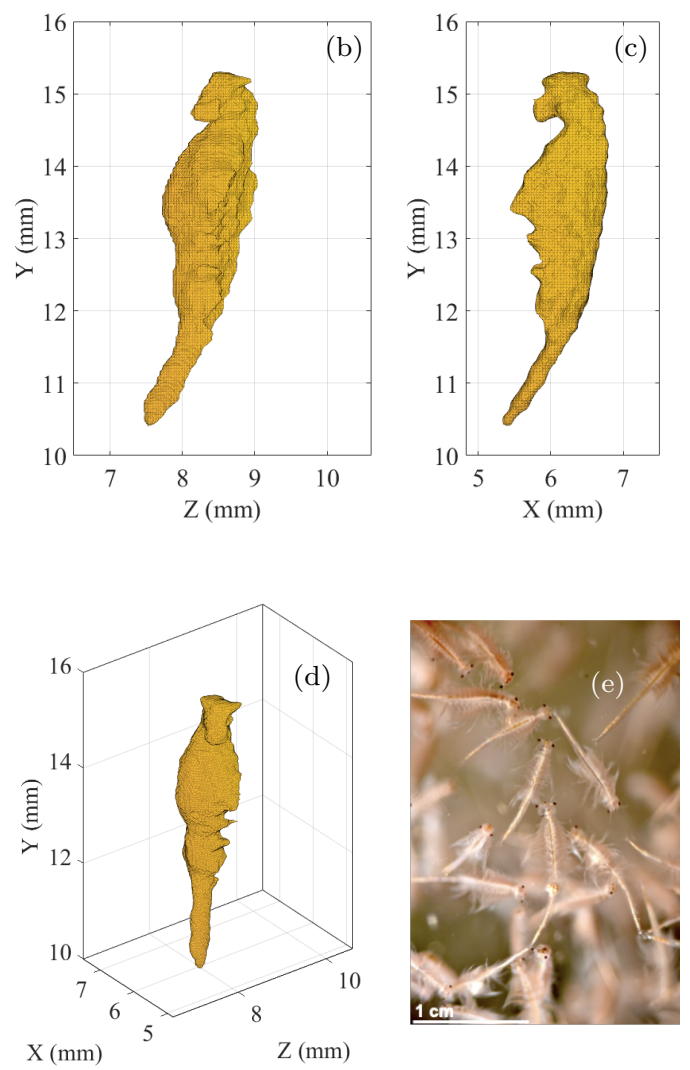

Fig. 5: Reconstructed swimmer bodies from an induced vertical migration. $\left(8 \times 10^{5}\right.$ animals per $\left.\mathrm{m}^{3}\right) .(\mathrm{a}) \mathrm{Re}^{-}$ construction of animals within the full imaging volume. The direction of the migration was in the positive $y$-direction. The number of swimmers and their 3D locations and orientations could be reconstructed with the scanning technique. (b)-(d) Images of a single reconstructed swimmer from the migration shown from different viewing angles. The 3D morphology of the swimmer was evident in the reconstruction, although some features of the shrimp were elongated along the scanning dimension. (e) A reference picture of Artemia salina.

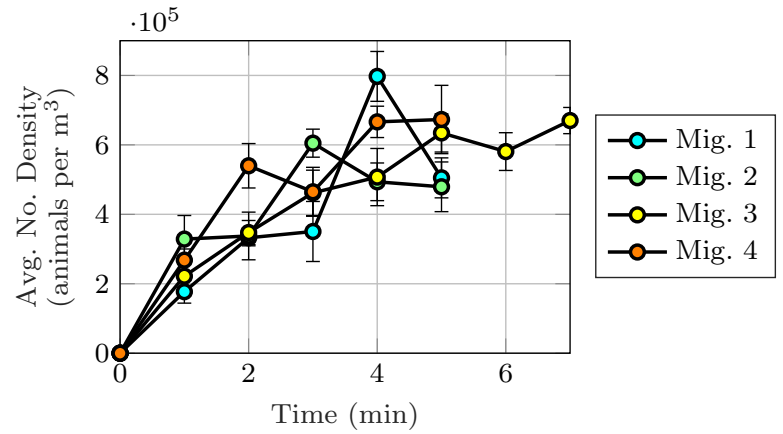

Fig. 6: Plot of the mean animal number density in the imaging volume over the duration of four induced vertical migrations. The upper-most point corresponds to the visualization shown in Figure 5.

Dabiri (2019), where a steady-state saturation in the number of shrimp was observed after one to two minutes, we observed a slow but continual growth in the number of shrimp in the frame, even up to four minutes. This slower migratory behavior may be attributed to differences in the age and health condition of the organisms tested presently or due to natural biological variability in the migratory behavior. For the present purposes, it is sufficient to note that the repeated measurements are qualitatively consistent. 


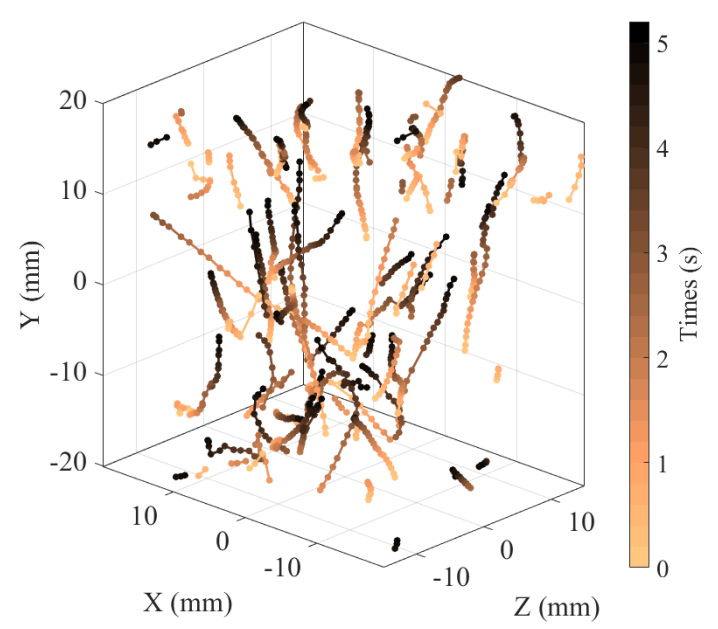

Fig. 7: Plot animal trajectories from Figure 5 over the $5 \mathrm{sec}$. duration of the scan indicated by the color change from copper to black.

Figure 7 shows the animal pathlines over the scanning period associated with the swimmers reconstructed in Figure 5. For the scanning frequency used here $\left(f_{s}=5 \mathrm{~Hz}\right)$, the displacement of individual shrimp between frames is typically a fraction of a body length. Because the displacement of each organism between frames is small relative to the inter-organism spacing, we can successfully track most organisms in these experiments with a nearest-neighbor search. More sophisticated particle tracking algorithms such as that of Ouellette et al. (2006) could improve the trajectory length and prediction.

\subsection{Velocity Measurements}

Corresponding contours of vertical velocity associated with the upward migration are shown in Figure 8 . Figure 8 a shows contours of the vertical velocity contours taken from a scan taken approximately 2 minutes into the migration. This scan was obtained closer to the beginning of the migration and contained fewer animals within the imaging volume than Figure 5a. Consequently, the technique was able to resolve downward projecting wakes from the individual swimmers. Figure $8 \mathrm{~b}$ shows contours of the vertical velocity associated with the scan shown in Figure 5a where the downward velocity was largest. A coherent downward motion of fluid through the aggregation was evident. This behavior was consistent with the observations of Houghton et al. (2018), who qualitatively visualized a similar coherent downward flow from vertical migration A. salina using planar laserinduced fluorescence. These measurements indicate that the technique was capable of quantitatively resolving the $3 \mathrm{D}$ velocity field in and around the swimming aggregation.

\section{Velocity Measurement Validation}

Due to the lack of a ground truth reference to validate the velocity measurements in the vertical migrations, this capability of the measurement system was assessed by using a controlled laminar jet flow. By evaluating the system against a laminar jet flow without an aggregation present, we were able to ensure that it could accurately resolve the three-component, three-dimensional velocity field. Imaging was conducted in a small $40 \times 40 \times 40 \mathrm{~cm}^{3}$ glass tank seeded with $100 \mu \mathrm{m}$ silver-coated glass spheres. A syringe pump provided a bulk flow of $21.50 \mathrm{~mL} / \mathrm{min}$ into a length of Tygon tubing with an elliptical cross section (equivalent diameter, $D_{e}=8.7 \mathrm{~mm}$ ) which exited as a laminar free jet flow of $R e_{D}=U_{b} D_{e} / \nu$, where $U_{b}$ was the bulk jet velocity, and $\nu$ was the water kinematic viscosity $\left(0.95 \mathrm{cSt}\right.$ at $\left.22^{\circ} \mathrm{C}\right)$. The finite eccentricity of the cross-section was due to plastic deformation of the tube wall prior to installation. Illumination was provided by a $532 \mathrm{~nm}$ laser with Gaussian beam shape. Here, the scanning speed, $u_{s}$, was 100 times larger than the jet's bulk velocity, 


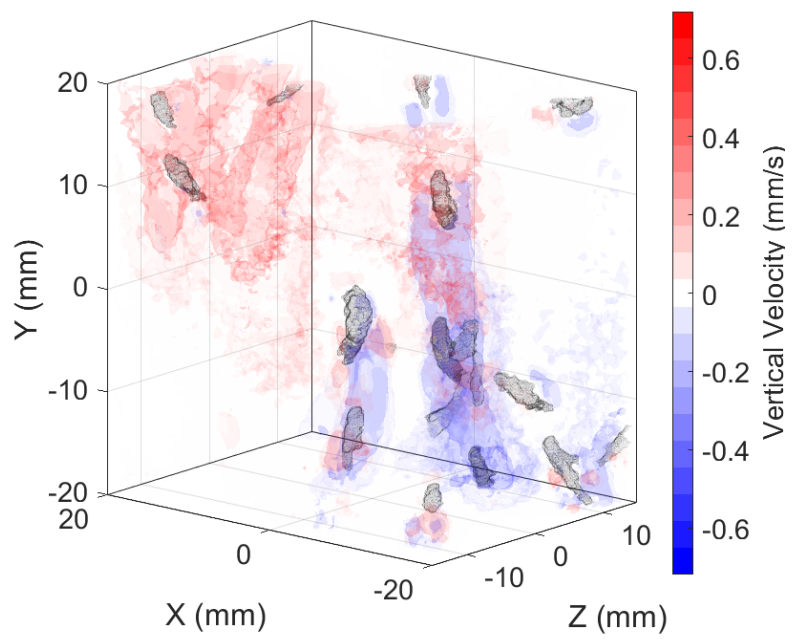

(a)

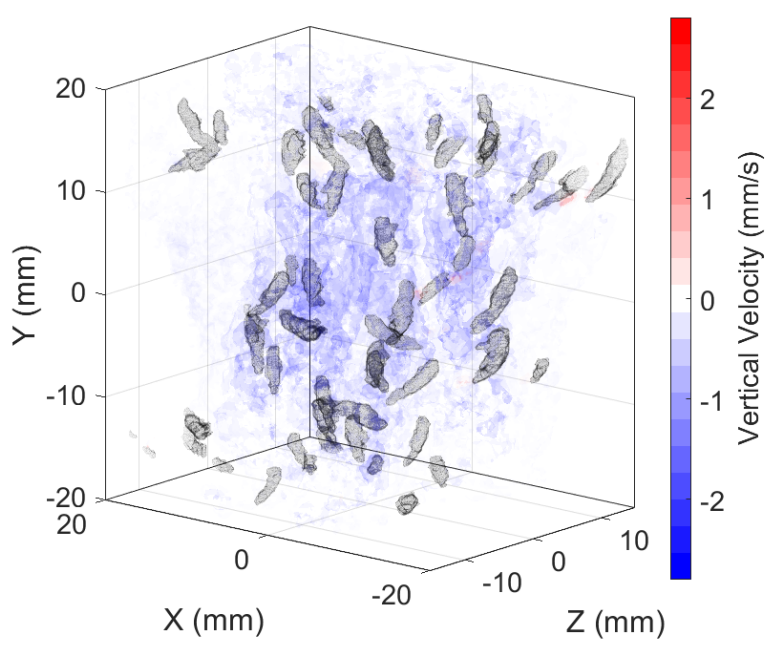

(b)

Fig. 8: Vertical velocity contours from snapshots taken during a single migration. Swimmer masks within the PIV field are shown in tan. Blue and red isosurfaces denote contours of negative and positive vertical velocity, respectfully. (a) Vertical velocity field 2 minutes into the migration. Downward velocity (blue) associated with the wakes of the swimming was resolved. (b) Vertical velocity field 4 minutes into the migration. Downward velocity (blue) contours associated with Figure 5a. Instead of individual wakes, a coherent downward motion was present through the migration.

which was sufficient to resolve the $3 \mathrm{D}$ particle positions with minimal error related to the finite scanning speed (Kozul et al., 2019). Imaging parameters and jet specifications are listen in Table 2 .

Measurements of the out-of-plane velocity component aligned with the scanning direction were verified by scanning the jet in two different orientations, as shown in Figure 9. In the first orientation (given by the blue jet in Figure 9a), the axis of the jet was parallel to the scanning direction such that the jet flow was normal to the imaging plane. An image slice of the jet in this configuration can be seen in Figure $9 \mathrm{~b}$ with the elliptical cross-section of the wall illuminated by the imaging sheet. Correspondingly, the tracer particle motion was primarily out of the page. In the second orientation (given by the green jet in Figure 9 a), the jet's axis was perpendicular to the scanning direction, and the tracer particle displacements were primarily contained within the same imaging plane. In the corresponding image slice (see Figure 95), the imaged cross-section of the tube instead appeared rectangular, and the fluid advection was from left to right. Resolving the flow in the first 


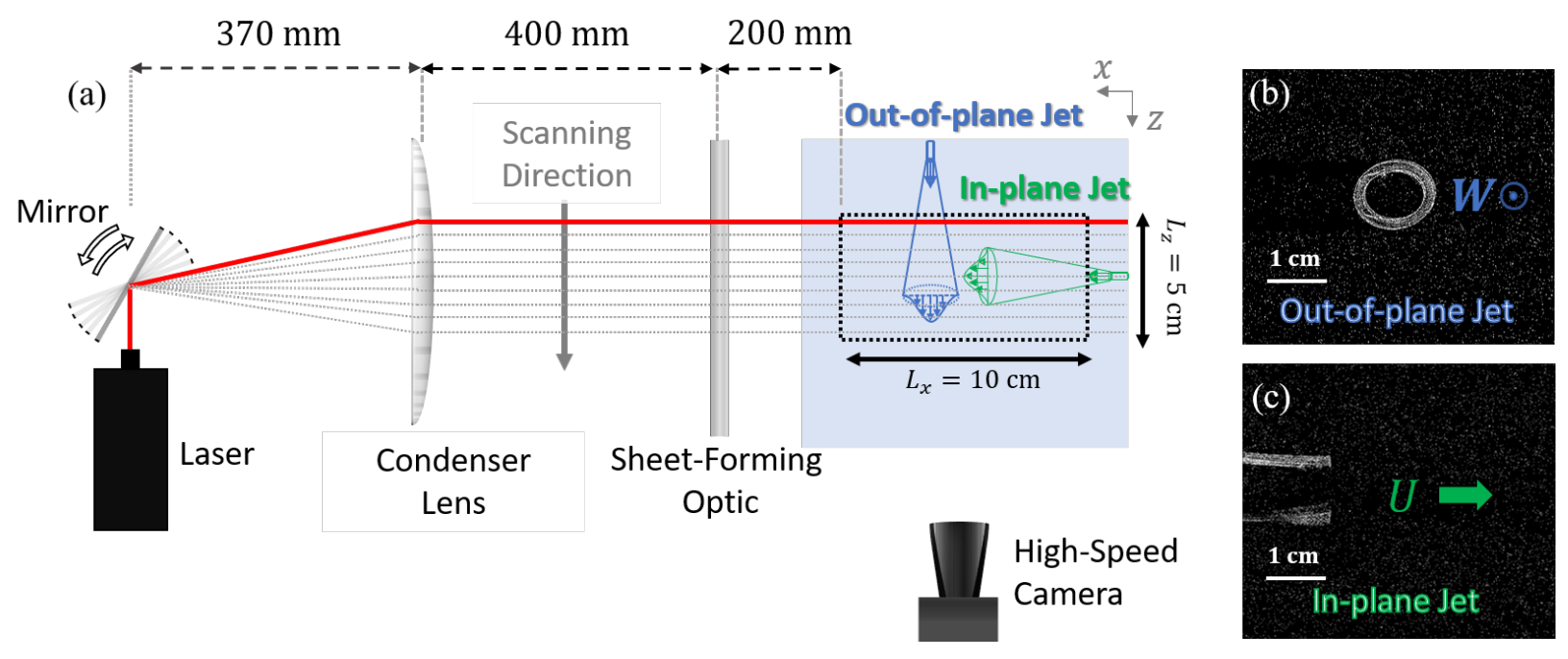

Fig. 9: Diagrams of the scanning system setup for flow velocity measurement validation. (a) Top view of the experimental setup. From this viewpoint, the scanning direction was parallel with the page's height, and the imaging planes were aligned with the page width and normal directions. The two orientations of the jet flow measured in this experiment corresponding to flow normal and parallel to the imaging plane are blue and green, respectively. (b) Image of tracer field and jet outlet in the out-of-plane orientation. The optical axis of the camera was parallel to the axis of the jet, and flow was out of the page. The elliptical cross-section seen in the image was due to plastic deformation of the tube prior to installation and was present in both configurations. (c) Image of tracer field and jet outlet as imaged from the in-plane orientation. The optical axis of the camera was perpendicular to the axis of the jet, and the flow advection was from left to right.

configuration depended on the ability of the technique to reconstruct tracer particle location along the scanning dimension. There, the velocity calculations correlated particles across different image sheets. Conversely, in the second configuration, velocity calculations were far less sensitive to the scanning effect as fluid motion was primarily contained within the image plane. Consequently, the fluid motion could still be determined without explicitly relying on the particles motions in adjacent image sheets similar to conventional 2D PIV. Hence, the in-plane jet measurement provided a ground truth reference for the out-of-plane measurements.

\begin{tabular}{|c|c|c|c|}
\hline & Parameter & Symbol & Value \\
\hline \multirow{8}{*}{ 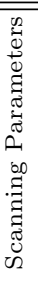 } & Field of View & 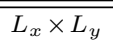 & $100 \times 100 \mathrm{~mm}^{2}$ \\
\hline & Depth of Field & $L_{z}$ & $47 \mathrm{~mm}$ \\
\hline & Approx. Voxel Size & 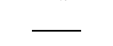 & $100 \times 100 \times 100 \mu \mathrm{m}^{3}$ \\
\hline & Image Acq. Rate & $f_{c}$ & $5,000 \mathrm{fps}$ \\
\hline & Scanning Freq. & $f_{s}$ & $10 \mathrm{~Hz}$ \\
\hline & Scanning Speed & $u_{s}$ & $50 \mathrm{~cm} \cdot \mathrm{s}^{-1}$ \\
\hline & $e^{-2}$ Sheet thickness & $h$ & $\approx 1 \mathrm{~mm}$ \\
\hline & Sheet step size & $\Delta z$ & $100 \mu \mathrm{m}$ \\
\hline \multirow{8}{*}{ 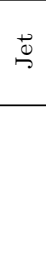 } & Bulk Velocity & $U_{b}$ & $5.4 \pm 0.1 \mathrm{~mm} / \mathrm{s}$ \\
\hline & Equivalent Diameter & $D_{e}$ & $8.7 \pm 0.2 \mathrm{~mm}$ \\
\hline & Aspect Ratio & $\overline{-}$ & 1.31 \\
\hline & Reynolds Number & $\operatorname{Re}_{D}$ & 50 \\
\hline & Non-dim Sheet Speed & $u_{s} / U_{b}$ & 92 \\
\hline & Non-dim Sheet Width & $h / \Delta z$ & 10 \\
\hline & No. laser sheets & $N$ & 475 \\
\hline & No. of scans & 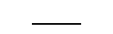 & 40 \\
\hline
\end{tabular}

Table 2: Imaging parameters for jet measurements

The two different configurations were evaluated by reorienting the jet within the tank while keeping the imaging system fixed. To evaluate the technique, we compared the maximum fluid velocities from each configu- 


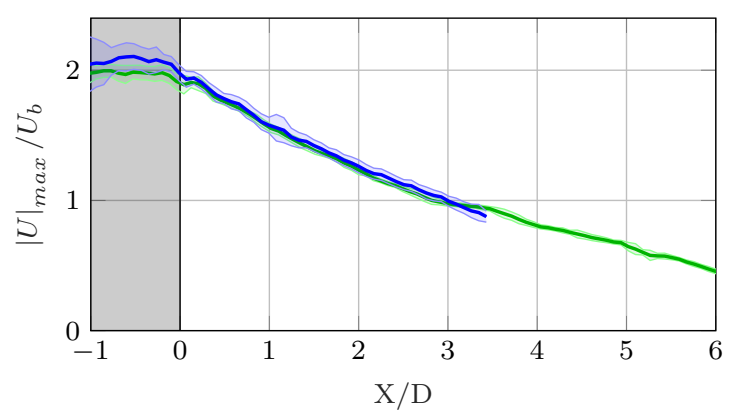

Fig. 10: Maximum fluid velocity $\left(U_{\max }\right)$ exiting the round jet as a function of distance from the jet exit. The gray area denotes measurements obtained inside the jet tube. (-): Out-of-plane jet (blue) where the fluid velocity was normal to the imaging plane. (-): In-plane jet (green) where the velocity was parallel to the imaging plane. Blue and green bands correspond to the standard deviation of the local velocity measurements in the out-of-plane and in-plane configurations, respectively.

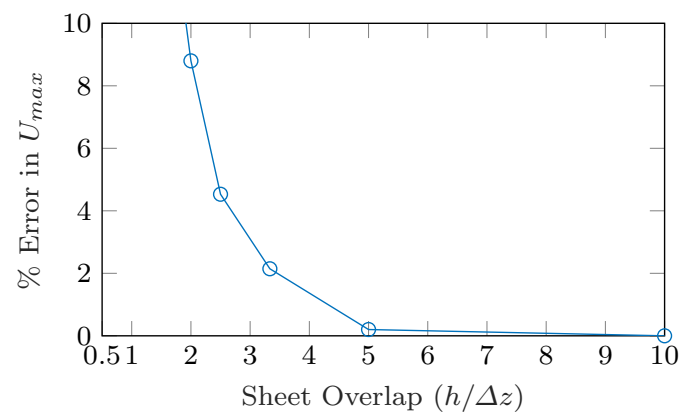

Fig. 11: Error in the max jet velocity calculation relative to $h / \Delta z=10$ as a function of sheet overlap.

ration as a function of distance from the jet exit, as shown in Figure 10. Because the jet tube is translucent, the technique is capable of measuring velocity inside the jet tube (shaded in gray), albeit with a slight difference between the two orientations. In this region, the out-of-plane orientation measures approximately $5 \%$ larger than its in-plane counterpart. Immediately outside the jet exit, there is excellent agreement between the two measurements over the extent of the domain. The distance over which data is reported for the out-of-plane jet is considerably shorter than the in-plane jet due to the depth of field being smaller than the image width. Importantly, this test indicates that the setup is capable of resolving velocities both parallel and normal to the imaging plane and is consistent with previously reported validations of scanning PIV (Brücker et al., 2013, Kozul et al., 2019).

Here, because of the fine sampling of the imaging volume, we were able to examine how the quality of the velocity calculations along the optical axis degraded with increased sheet spacing. For the experiment conducted here, the step size between consecutive images was approximately the voxel size which corresponded to a $90 \%$ overlap between adjacent sheets (i.e., $h / \Delta z=10$ ). Here, we artificially increased the step size of our data set by first down-sampling the image volumes from full-resolution scans and then re-interpolating via tri-spline the new images back to the full resolution. The images were then processed with the same cross - correlation algorithm and compared with the full-resolution result $(h / \Delta z=10)$. Figure 11 shows how the mean difference between the max velocity calculation shown in Figure 10 varied as the effective step size between laser sheets was increased. Importantly, even when the data was down-sampled by a factor of $2(h / \Delta z=5)$, there was a negligible change in the measured maximum jet velocity over the domain. Even downsampling the data by a factor of $3(h / \Delta z=3.33)$, yielded a mean error of approximately $2 \%$ over the imaging domain compared to the full resolution measurement. Above this range, the error began to increase sharply as the spacing approached the sheet width. These empirical results were consistent with previous findings from comparable numerical investigations including Kozul et al. (2019) who found a sheet overlap of $h / \Delta z=5$ to be sufficiently resolved 
for particle tracking and Lawson and Dawson (2014) who found $h / \Delta z=3-4$ to be optimal for single camera measurements.

\section{Discussion and Conclusions}

A 3D scanning velocimetry system for 3D-3C velocity measurements and particle aggregation reconstruction was demonstrated using an induced vertical migration of $A$. salina. The technique successfully reconstructed the swimmer bodies and their 3D configurations at animal number densities at the upper bound of those found in previous laboratory migration experiments $\left(8 \times 10^{5}\right.$ animal per $\left.\mathrm{m}^{3}\right)$, a task that had not been accomplished with previous methods. This capability will allow for more direct studies of the flow-structure interactions that enable individual animal wakes to coalesce into larger-scale flows. The success of this technique at these animal number densities suggest that it could have broader applications in the study of flows with dispersed particles. The animal volume fractions measured in this study, $\Phi_{v} \leq 1.7 \pm 0.24 \times 10^{-3}$, encompass the range of volume fractions $\left(10^{-6} \leq \Phi_{v} \leq 10^{-3}\right)$ over which two-way coupling is exhibited between turbulence and dispersed particles (Elghobashi, 1994). This capability suggests that scanning techniques could be a robust tool for studying this coupling in turbulent flows with translucent or transparent particles, such as bubbles and droplets.

The most notable challenges for this system included the trade-offs between the temporal resolution of the flow field, illumination of the images, and the resolvable depth of field. The achievable depth of field in the present design was primarily constrained by the power of the laser. Increasing the depth of field to keep all of the scans in sharp focus required significantly reducing the image illumination due to compounding effects of shrinking the lens aperture and increasing the camera frame rate. In the case of the former, reducing the aperture caused a quadratic reduction in the light intensity for a linear increase in the depth of field.

Future implementations of this technique can employ a telecentric lens on the high-speed camera to ensure a constant magnification throughout the entire image volume, eliminating any parallax. Additionally, this lens type will also allow for a larger usable depth of field for a given aperture due to the symmetric image blurring. Similarly, incorporating a scanning lens into the setup could significantly improve the temporal capabilities of the scanning system. A scanning lens would allow the location of the focal plane to be adjusted over distances comparable to the field of view at bandwidths exceeding the scanning frequencies. By synchronizing the focal distance to the laser sheet location, the depth of field can be reduced to the thickness of the laser sheet and individual images can be captured by the high-speed camera using a much larger aperture. This modification would allow for significantly greater illumination of the camera sensor than the current implementation where the focal plane is static, and the entire scanning distance must be contained within depth of field.

Lastly, because the technique relies on a single high-speed camera, it is compatible with many existing underwater imaging systems such as the diver operated self-contained underwater velocimetry apparatus (SCUVA) (Katija and Dabiri, 2008) or remotely operated DeepPIV (Katija et al., 2020, 2017). Adapting this technique for field deployment could enable 3D-3C velocity measurements of various environmental and biological flows that have traditionally been limited to $2 \mathrm{D}$ observations. Similar to the A. salina, there are numerous marine organism whose feeding and swimming are potentially observable with this technique, including salps, jellyfish, siphonophores, and ctenophores. The ability to image the 3D flow in and around these organisms could provide numerous biological and fluid mechanical insights.

Acknowledgements The authors would like to thank Prof. Christian Franck for supplying the basis for the cross-correlation algorithm used in this study. This work was supported by the U.S. National Science Foundation Grant, under Award Number 1510607 and the Gordon and Betty Moore Foundation.

\section{References}

Adhikari D, Longmire EK (2012) Visual hull method for tomographic PIV measurement of flow around moving objects. Experiments in Fluids 53(4):943-964, DOI 10.1007/s00348-012-1338-9

Adhikari D, Webster DR, Yen J (2016) Portable tomographic PIV measurements of swimming shelled Antarctic pteropods. Exp Fluids 57:180, DOI 10.1007/s00348-016-2269-7

Aliseda A, Heindel TJ (2021) X-Ray Flow Visualization in Multiphase Flows. Annual Review of Fluid Mechanics 53(1):543-567, DOI 10.1146/annurev-fluid-010719-060201 
Balachandar S, Eaton JK (2010) Turbulent Dispersed Multiphase Flow. Annu Rev Fluid Mech 42:111-133, DOI 10.1146/annurev.fluid.010908.165243

Bar-Kochba E, Toyjanova J, Andrews E, Kim KS, Franck C (2015) A Fast Iterative Digital Volume Correlation Algorithm for Large Deformations. Experimental Mechanics 55:261-274, DOI 10.1007/s11340-014-9874-2

Biggs DSC, Andrews M (1997) Acceleration of iterative image restoration algorithms. Applied Optics 36(8):1766, DOI 10.1364/ao.36.001766

Bourgoin M, Xu H (2014) Focus on dynamics of particles in turbulence. New Journal of Physics 16, DOI $10.1088 / 1367-2630 / 16 / 8 / 085010$

Brücker C, Hess D, Kitzhofer J (2013) Single-view volumetric PIV via high-resolution scanning, isotropic voxel restructuring and 3D least-squares matching (3D-LSM). Measurement Science and Technology 24(2):024001, DOI 10.1088/0957-0233/24/2/024001

Elghobashi S (1994) On predicting particle-laden turbulent flows. Applied Scientific Research 52(4):309-329, DOI 10.1007/BF00936835

Gemmell BJ, Sheng J, Buskey EJ (2013) Compensatory escape mechanism at low Reynolds number. Proceedings of the National Academy of Sciences 110(12):4661-4666, DOI 10.1073/PNAS.1212148110

Gemmell BJ, Colin SP, Costello JH, Sutherland KR (2019) A ctenophore (comb jelly) employs vortex rebound dynamics and outperforms other gelatinous swimmers. Royal Society Open Science 6(3):181615, DOI 10. $1098 /$ rsos. 181615

Houghton IA, Dabiri JO (2019) Alleviation of hypoxia by biologically generated mixing in a stratified water column. Limnology and Oceanography 64(5):2161-2171, DOI 10.1002/lno.11176

Houghton IA, Koseff JR, Monismith SG, Dabiri JO (2018) Vertically migrating swimmers generate aggregationscale eddies in a stratified column. Nature DOI 10.1038/s41586-018-0044-z

Hoyer K, Holzner M, Lüthi B, Guala M, Liberzon A, Kinzelbach W (2005) 3D scanning particle tracking velocimetry. Experiments in Fluids 39(5):923-934, DOI 10.1007/s00348-005-0031-7

Jumars PA, Trowbridge JH, Boss E, Karp-Boss L (2009) Turbulence-plankton interactions: a new cartoon. Marine Ecology 30(2):133-150, DOI 10.1111/j.1439-0485.2009.00288.x

Katija K, Dabiri JO (2008) In situ field measurements of aquatic animal-fluid interactions using a Self-Contained Underwater Velocimetry Apparatus (SCUVA). Limnology and Oceanography: Methods 6(4):162-171, DOI 10.4319/lom.2008.6.162

Katija K, Sherlock RE, Sherman AD, Robison BH (2017) New technology reveals the role of giant larvaceans in oceanic carbon cycling. Science Advances 3(5):e1602374, DOI 10.1126/sciadv.1602374

Katija K, Troni G, Daniels J, Lance K, Sherlock RE, Sherman AD, Robison BH (2020) Revealing enigmatic mucus structures in the deep sea using DeepPIV. Nature pp 1-5, DOI 10.1038/s41586-020-2345-2

Khalitov DA, Longmire EK (2002) Simultaneous two-phase PIV by two-parameter phase discrimination. Experiments in Fluids 32(2):252-268, DOI 10.1007/s003480100356

Kozul M, Koothur V, Worth NA, Dawson JR (2019) A scanning particle tracking velocimetry technique for high-Reynolds number turbulent flows. Experiments in Fluids 60:137, DOI 10.1007/s00348-019-2777-3

Kunze E (2019) Biologically generated mixing in the ocean. DOI 10.1146/annurev-marine-010318-095047

Lawson JM, Dawson JR (2014) A scanning PIV method for fine-scale turbulence measurements. Exp Fluids $55: 1857$, DOI $10.1007 / \mathrm{s} 00348-014-1857-7$

Mendelson L, Techet AH (2015) Quantitative wake analysis of a freely swimming fish using 3D synthetic aperture PIV. Experiments in Fluids 56(7):1-19, DOI 10.1007/s00348-015-2003-x

Mendelson L, Techet AH (2018) Multi-camera volumetric PIV for the study of jumping fish. Experiments in Fluids 59(1):10, DOI 10.1007/s00348-017-2468-x

More RV, Ardekani AM (2021) Hydrodynamic interactions between swimming microorganisms in a linearly density stratified fluid. Physical Review E 103(1):013109, DOI 10.1103/PhysRevE.103.013109

Ni R, Kramel S, Ouellette NT, Voth GA (2015) Measurements of the coupling between the tumbling of rods and the velocity gradient tensor in turbulence. Journal of Fluid Mechanics 766:202-225, DOI 10.1017/jfm.2015.16, 1411.6072

Nogueira J, Lecuona A, Rodrìguez PA, Alfaro JA, Acosta A (2005) Limits on the resolution of correlation PIV iterative methods. Practical implementation and design of weighting functions. Experiments in Fluids 39(2):314-321, DOI 10.1007/s00348-005-1017-1

Noss C, Lorke A (2014) Direct observation of biomixing by vertically migrating zooplankton. Limnology and Oceanography 59(3):724-732, DOI 10.4319/lo.2014.59.3.0724

Otsu N (1979) Threshold selection method from gray-level histograms. IEEE Transactions on Systems, Man, and Cybernetics SMC-9(1):62-66, DOI 10.1109/tsmc.1979.4310076 
Ouellette NT, Xu H, Bodenschatz E (2006) A quantitative study of three-dimensional Lagrangian particle tracking algorithms. Experiments in Fluids 40(2):301-313, DOI 10.1007/s00348-005-0068-7

Ouillon R, Houghton IA, Dabiri JO, Meiburg E (2020) Active swimmers interacting with stratified fluids during collective vertical migration. Journal of Fluid Mechanics 902, DOI 10.1017/jfm.2020.618

Pereira F, Gharib M (2002) Defocusing digital particle image velocimetry and the three-dimensional characterization of two-phase flows. Measurement Science and Technology 13(5):683-694, DOI 10.1088/0957-0233/ $13 / 5 / 305$

Rensen J, Luther S, Lohse D (2005) The effect of bubbles on developed turbulence. Journal of Fluid Mechanics 538:153-187, DOI 10.1017/S0022112005005276

Risso F (2018) Agitation, Mixing, and Transfers Induced by Bubbles. Annual Review of Fluid Mechanics 50(1):25-48, DOI 10.1146/annurev-fluid-122316-045003

Schanz D, Gesemann S, Schröder A (2016) Shake-The-Box: Lagrangian particle tracking at high particle image densities. Experiments in Fluids 57(5):70, DOI 10.1007/s00348-016-2157-1

Schrijer FF, Scarano F (2008) Effect of predictor-corrector filtering on the stability and spatial resolution of iterative PIV interrogation. Experiments in Fluids 45(5):927-941, DOI 10.1007/s00348-008-0511-7

Simoncelli S, Thackeray SJ, Wain DJ (2018) On biogenic turbulence production and mixing from vertically migrating zooplankton in lakes. Aquatic Sciences 80(4), DOI 10.1007/s00027-018-0586-z

Su I, Qin Z, Saraceno T, Krell A, Mühlethaler R, Bisshop A, Buehler MJ (2018) Imaging and analysis of a three-dimensional spider web architecture. Journal of the Royal Society Interface 15(146), DOI 10.1098/rsif. 2018.0193

Tan ZP, Alarcon R, Allen J, Thurow BS, Moss A (2020) Development of a high-speed plenoptic imaging system and its application to marine biology PIV. Measurement Science and Technology 31(5):054005, DOI $10.1088 / 1361-6501 / \mathrm{ab} 553 \mathrm{c}$

Troutman VA, Dabiri JO (2018) Single-camera three-dimensional tracking of natural particulate and zooplankton. Measurement Science and Technology 29(7):075401, DOI 10.1088/1361-6501/aac15a

Ullah A, Masuk M, Salibindla A, Ni R (2019) International Journal of Multiphase Flow A robust virtual-camera $3 \mathrm{D}$ shape reconstruction of deforming bubbles/droplets with additional physical constraints. International Journal of Multiphase Flow 120:103088, DOI 10.1016/j.ijmultiphaseflow.2019.103088

Wang S, Ardekani A (2012) Inertial squirmer. Physics of Fluids 24(10):101902, DOI 10.1063/1.4758304

Wang S, Ardekani AM (2015) Biogenic mixing induced by intermediate Reynolds number swimming in stratified fluids. Scientific Reports 5(1):17448, DOI 10.1038/srep17448

Westerweel J, Scarano F (2005) Universal outlier detection for PIV data. Experiments in Fluids 39(6):10961100, DOI 10.1007/s00348-005-0016-6

Wilhelmus MM, Dabiri JO (2014) Observations of large-scale fluid transport by laser-guided plankton aggregations. Physics of Fluids 26(10):1-12, DOI 10.1063/1.4895655 